\title{
Diseño de un Sistema de Gestión de Inocuidad Alimentaria Bajo la Norma ISO 22000:2005 para Una Planta Procesadora de Quinua
}

\author{
María José Andrade Albán, MsC \\ Cristina Valeria Calderón Vallejo, MsC \\ María de los Ángeles Rodríguez Cevallos, MsC \\ Juan Carlos Naranjo Herrera, MsC
}

Docentes de Tiempo Completo de la Facultad de Salud Pública Escuela

Superior Politécnica de Chimborazo, Ecuador

Armando Vinicio Paredes Peralta, $\mathrm{MsC}$

Fredy Patricio Erazo Rodríguez, $\mathrm{MsC}$

Docente de Tiempo Completo de la Facultad de Ciencias Pecuarias Escuela

Superior Politécnica de Chimborazo, Ecuador

Doi:10.19044/esj.2019.v15n21p317 URL:http://dx.doi.org/10.19044/esj.2019.v15n21p317

\section{Resumen}

La presente investigación tiene como objetivo diseñar un sistema de gestión de inocuidad alimentaria bajo la Norma ISO 22000:2005 para la planta procesadora de quinua de la Corporación de Productores y Comercializadores Orgánicos Bio Taita Chimborazo (COPROBICH), mediante la integración de los diferentes manuales, procedimientos y aplicaciones que plasmen las actividades llevadas a cabo diariamente en la planta. La implantación de este sistema constituirá una llave que abrirá la puerta a la posibilidad de ingresar de manera efectiva y eficiente a mercados internacionales, cuyos parámetros de exigencia son ciertamente altos, garantizando la producción de alimentos inocuos. El desarrollo de este trabajo partió con una investigación de campo, puesto que se llevaron a cabo auditorías que permitieron identificar los agentes causales que generan bajos estándares de seguridad alimentaria. Asimismo se realizó una evaluación diagnóstica de la situación actual de la empresa, para lo cual se aplicó un test basado en la Norma Internacional ISO 22000:2005, cuyo resultado reflejó un porcentaje de cumplimiento igual a 12 . Posteriormente se estructuró el sistema documental de acuerdo a los programas prerrequisitos que establece la Norma Internacional ISO 22000:2005; se perfiló igualmente el Plan HACCP a partir de la formación de un equipo de inocuidad y el análisis de peligros y puntos críticos de control de cada una de las etapas que comprende el procesamiento y comercialización de 
quinua orgánica, con lo cual se incrementó a 52\% el cumplimiento del test aplicado.

Palabras clave: ISO 22000:2005, Inocuidad Alimentaria, Punto Crítico de Control, COPROBICH, Peligro de Inocuidad

\title{
Design of a Food Safety Management System Based on the ISO 22000:2005 for the Processing Quinoa Factory of Bio Taita Chimborazo Organic Marketers and Producers Corporation, Coprobich
}

\author{
María José Andrade Albán, MsC \\ Cristina Valeria Calderón Vallejo, $M \mathrm{MS}$ \\ María de los Ángeles Rodríguez Cevallos, MsC \\ Juan Carlos Naranjo Herrera, MsC \\ Docentes de Tiempo Completo de la Facultad de Salud Pública Escuela \\ Superior Politécnica de Chimborazo, Ecuador \\ Armando Vinicio Paredes Peralta, MsC \\ Fredy Patricio Erazo Rodríguez, $\mathrm{MsC}$ \\ Docente de Tiempo Completo de la Facultad de Ciencias Pecuarias Escuela \\ Superior Politécnica de Chimborazo, Ecuador
}

\section{Abstract}

This paper focuses on designing a food safety management system under the ISO 22000: 2005 Standard for the quinoa processing plant of the Organic Taita Chimborazo Organic Producers and Marketers Corporation. This was achieved through the integration of the different manuals, procedures, and applications that reflect the activities carried out daily in the plant. The implementation of this system will serve as a key that will open the possibility of effectively and efficiently entering international markets, whose parameters of demand are certainly high, guaranteeing the production of safe food. The development of this study started with a field investigation. This is because audits were carried out that help to identify the causal agents generating low standards of food security. Likewise, a diagnostic evaluation of the current situation of the company was carried out. In this case, a test was applied based on the International Standard ISO 22000: 2005. The result reflected a fulfillment percentage equal to 12 . Afterward, a documental system 
was structured according to the prerequirement programs that the ISO 22000:2005 International Standard establishes. HACCP plan was outlined starting with the conformation of a safety group, the hazard analysis, critical control points of one and each of the stages that the procedure includes, and organic quinoa commercialization through which the test applied experience a significant increase at a $52 \%$ of the fulfillment.

Keywords: ISO 22000: 2005, Food Safety, Critical Control Point, COPROBICH, Safety hazard

\section{Introducción}

Los Sistemas de Gestión de Calidad apuntan a garantizar de manera continua y ubicua un cumplimiento efectivo y eficiente de las tareas y responsabilidades (Van, 2005). Las normas o estándares internacionales que establecen las pautas para implantar en una organización diferentes sistemas de gestión (conocidas en inglés por Management System Standard) son el resultado de la normalización o estandarización, que se podría definir, de forma genérica, como la actividad encaminada a poner orden en aplicaciones repetitivas que se desarrollan en el ámbito de la industria, tecnología, ciencia y la economía. (Wong López, 2014)

De acuerdo a Martínez (2010), la industria alimenticia no ha sido la excepción en la globalización, razón por la cual ha tenido que adaptarse a los cambios en las preferencias de los consumidores, las cuales cambian de acuerdo a gustos, precios e higiene. Este último aspecto es de gran importancia para los consumidores, ya que de ahí se pueden generar una serie de problemas que pueden llegar a afectar el desarrollo de sus actividades, así como sus ingresos y especialmente su salud.

Es evidente que la globalización es el motor que impulsa a las organizaciones a esforzarse por exceder las expectativas de sus clientes, para con ello lograr la competitividad y reconocimiento necesario. Por ello, la International Standard Organization (ISO) inició un largo trabajo para desarrollar un conjunto de normas para el sector manufacturero, del comercio y la comunicación; las cuales pretenden obtener clientes más satisfechos y una empresa más productiva, al mismo tiempo que ofrece la oportunidad de mejorar continuamente y lograr una carta de presentación excelente (Ramírez \& Sánchez, 2006).

Por su parte, La Ley Orgánica del Régimen de Soberanía Alimentaria menciona el derecho que tienen las personas a acceder a alimentos nutritivos y culturalmente adecuados que hayan sido producidos de forma sostenible y ecológica, por lo que la Corporación de Productores y Comercializadores Orgánicos Bio Taita Chimborazo (COPROBICH), nace como una iniciativa para fortalecer y apoyar el trabajo de varias familias indígenas de la provincia 
de Chimborazo que han dedicado su trabajo a la siembra y cultivo de quinua.

Así pues, COPROBICH nació como iniciativa de la Fundación Escuelas Radiofónicas Populares del Ecuador para impulsar la distribución y comercialización de los productos orgánicos producidos en esta zona de la provincia de Chimborazo. La organización está legalmente reconocida, es de derecho privado, soberana, sin fines de lucro; su principio es de servicio y beneficio social para sus socios que son indígenas de cantones como Riobamba, Colta, Guamote, Guano y Penipe que entregan sus productos orgánicos para la comercialización y transformación. Por tal motivo, el desarrollo de este estudio incide directamente en dichas familias al permitir que la planta que acopia, procesa y comercializa su producto pueda contar con un Sistema de Gestión de Inocuidad Alimentaria, que les conceda la oportunidad de acceder y extenderse a otros mercados extranjeros cuyos parámetros de exigencia son ciertamente altos, garantizando la producción de alimentos inocuos.

En este sentido, la investigación tiene como objetivo diseñar un Sistema de Gestión de Inocuidad Alimentaria bajo la Norma ISO 22000:2005 para la planta procesadora de quinua de la Corporación de Productores y Comercializadores Orgánicos Bio Taita Chimborazo (COPROBICH).

\section{Metodología}

\section{Modalidad básica de la investigación}

La modalidad de trabajo se basó en una investigación de campo basada en los siguientes aspectos:

- Información que provino entre otras de la aplicación de cuestionarios y observaciones realizadas durante auditorías internas dentro de la organización. Se aplicó un check list basado en la Norma ISO 22000:2005 para evaluar la situación actual de la planta de procesamiento y poder definir las estrategias que se aplicarán para ayudar a COPROBICH a mejorar los aspectos que influyen directamente en la inocuidad de la quinua que se procesa.

- En este tipo de investigación se obtuvo la información directamente de la realidad en que se encuentra el objeto de estudio (planta de procesamiento de quinua), por lo tanto, implicó observación directa por parte del investigador para corroborar el nivel de cumplimiento de las normativas vigentes y poder intervenir.

\section{Nivel o tipo de investigación}

Se desarrolló una investigación de campo ya que se llevaron a cabo auditorías internas, con el fin de describir de qué modo o cuáles son los agentes causales que generan bajos estándares de seguridad alimentaria relacionada a la inocuidad dentro de COPROBICH. Así también se realizó un diagnóstico 
de las necesidades de capacitación y problemas de ejecución a efectos de implementar los conocimientos. La investigación fue igualmente de tipo exploratoria, ya que esta consiste en la búsqueda de datos que se suponen están relacionados a algún hecho. El resultado de esta exploración entregó información y datos que al ser clasificados, ordenados, analizados e interpretados, permitieron obtener una visión general de la realidad de la planta.

\section{Población y muestra}

La población estuvo conformada por 1632 familias, provenientes de 86 comunidades de la provincia de Chimborazo que son quienes pertenecen a la Corporación de Productores y Comercializadores Orgánicos Bio Taita Chimborazo(COPROBICH). No se realiza muestreo por ser una población pequeña y que entrega su producto de acuerdo a su producción agrícola, de modo que se trabajará con todos los integrantes de la población.

\section{Plan de recolección de información}

- Revisión bibliográfica: se revisaron los requisitos y disposiciones establecidas para la elaboración de un plan para la aplicación de un Sistema de Gestión de Inocuidad Alimentaria basado en la Norma ISO 22000:2005.

- Compilación de datos: se reunió información relevante y representativa acerca del volumen de exportación actual de la planta procesadora de quinua.

- Diagnóstico: para realizar el análisis de la situación actual se debió realizar una Auditoria de diagnóstico ISO 22000:2005 en la planta procesadora de quinua.

\section{Plan de procesamiento de la información}

- Revisión bibliográfica: revisión de libros, revistas, estudios científicos, documentos técnicos, artículos técnicos, normativas, leyes y reglamentos.

- Compilación de datos bibliográficos: luego de haber revisado la bibliografía existente sobre el tema, se seleccionaron los formatos que servirán para el desarrollo de los procedimientos (Objetivo, Alcance, Responsables, Procedimientos, Frecuencia de monitoreo, Registros y Firmas) y POES (Procedimientos Operativos Estandarizados de Saneamiento) .

- Herramientas para el procesamiento de datos: se emplearon programas informáticos como Excel, Word y Power Point.

- Análisis e interpretación de resultados: la interpretación de resultados se realizó con apoyo en la Norma Internacional ISO 22000:2005. 


\section{Resultados}

Se presenta un consolidado de la evaluación de diagnóstico en cumplimiento de la Norma Internacional ISO 22000:2005, de acuerdo al cuestionario de diagnóstico elaborado para COPROBICH

Tabla 1. Resultados de auditoría de diagnóstico (Andrade et al., 2017)

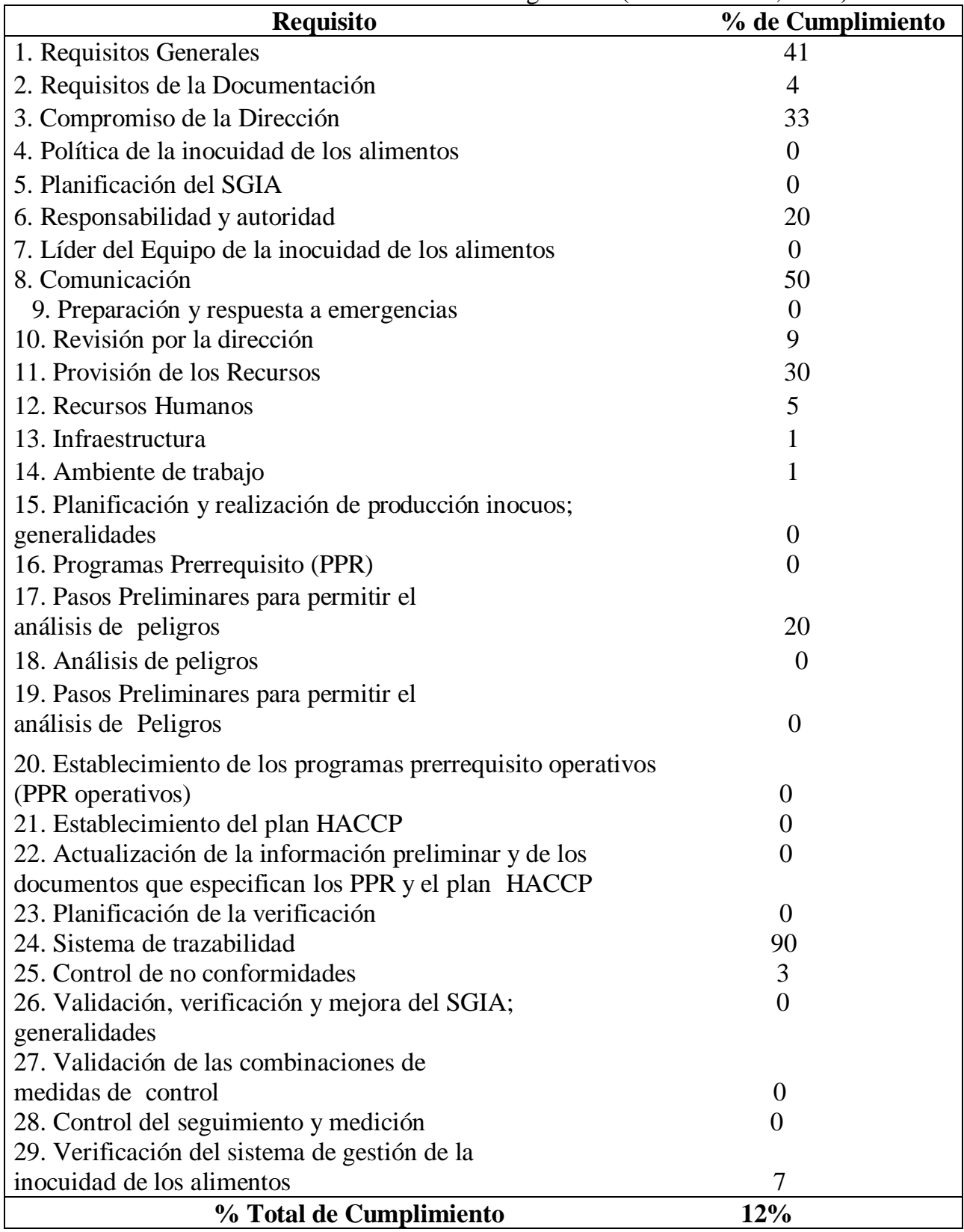

Como se puede observar en la Tabla 1 el porcentaje de cumplimiento en cuanto a las conformidades en la auditoria interna basada en la Norma ISO 
22000:2005 fue del $12 \%$. Por lo tanto, el trabajo partirá desde la planeación y aplicación de estrategias para mejorar la situación de la planta en cuanto a sus no conformidades.

\section{Resultados de la Auditoria de Diagnóstico \\ DPuntajes máximos a obtener $\quad$ Puntaje Obtenido}

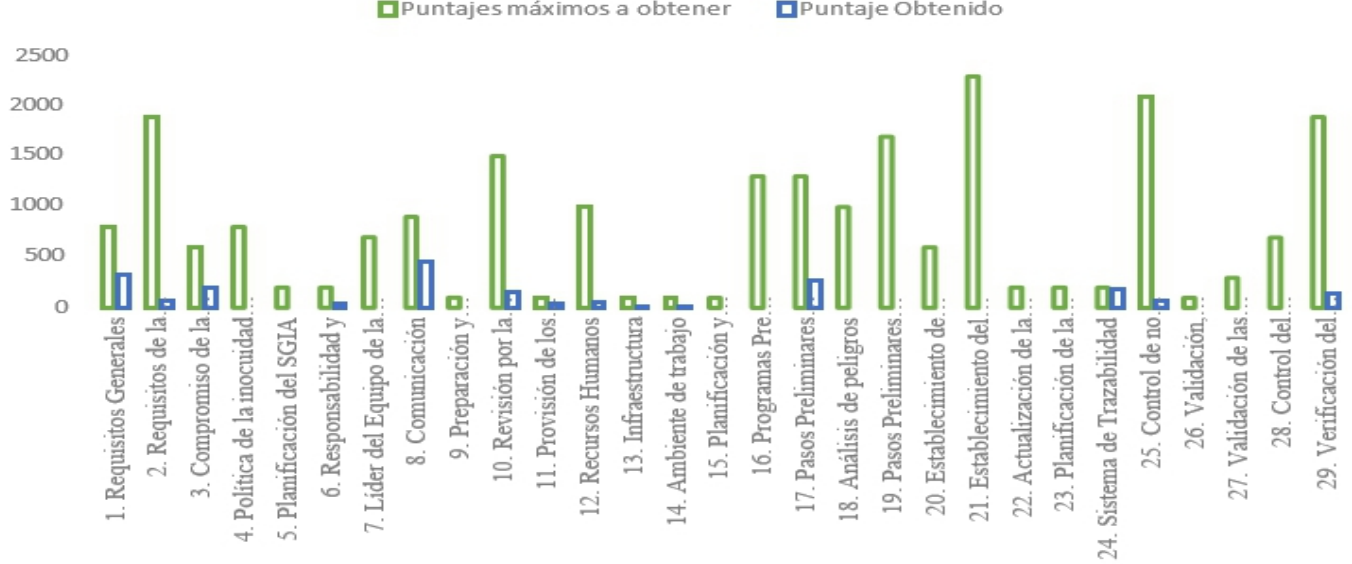

Figura 1. Esquema comparativo de los resultados de auditoría de diagnóstico

En la Figura 1 se aprecia un esquema comparativo de los puntajes que representan cada uno de los aspectos que se verificaron dentro de la Auditoría de diagnóstico y el valor numérico alcanzado por parte de la organización.

La organización carece de un Sistema de Gestión de Inocuidad Alimentaria (SGIA), razón por la cual no existe documentación en donde se especifiquen los productos o procesos y los lugares de producción que trabajen bajo un SGIA; se debe resaltar que COPROBICH elabora un solo producto. Los peligros considerados dentro del proceso de elaboración de quinua procesada se controlan de forma empírica, no existen procedimientos que estandaricen este control ni tampoco registros del mismo. El transporte y el control de plagas constituyen servicios que se contratan externamente, los cuales están controlados para asegurarse que cumplan con las exigencias de los países de destino. Se evidencia registros de estos controles.

COPROBICH carece igualmente de políticas de inocuidad. Sus procedimientos y registros no se encuentran documentados de acuerdo a un SGIA, por lo que tampoco existe un control de documentación ni un esquema de aprobación y actualización para los mismos. No obstante, la organización tiene definidas su política, misión y visión institucional de manera eficiente.

Un aspecto muy relevante es el compromiso y el interés que mostró la alta dirección para el diseño e implementación de un SGIA, garantizando así la disponibilidad de recursos para la implantación y mantenimiento del sistema.

Respecto al apartado que se refiere a responsabilidad y autoridad la organización tiene definidas las responsabilidades y autoridades a nivel 
directivo y a nivel ejecutivo. Cabe resaltar que dentro del nivel operativo los 11 operarios que rotan constantemente no están definidos; tampoco se ha designado un líder de inocuidad.

La comunicación de la organización internamente es limitada; sin embargo, la comunicación con sus clientes externos es muy eficaz, siendo en su totalidad extranjeros con los que se realiza contacto a través de redes telefónicas y vía on-line. Este contacto lo realiza el jefe de ventas de la organización quien presenta los registros que evidencian esto. COPROBICH no cuenta con un procedimiento donde se indique como se deben gestionar las potenciales situaciones de emergencia y accidentes que pueden afectar la inocuidad de los alimentos.

La organización fue recientemente inspeccionada por la Agencia Nación de Regulación, Control y Vigilancia Sanitaria (ARCSA), resultados que incentivaron a la alta dirección a buscar los recursos que permitan el diseño e implementación de un sistema de inocuidad y el desarrollo de programas de capacitación al personal en temas relacionados con la inocuidad de los alimentos, ya que no hay evidencia de que se evalúen y se fortalezcan las competencias del personal que labora en la organización.

En lo que se refiere al ambiente de trabajo la alta dirección proporciona los recursos adecuados para establecer y mantener el ambiente de trabajo; sin embargo, el personal por falta de práctica y de conocimiento subutiliza los recursos entregados.

No se evidencia la implementación de ningún PPR o PPR operativo que sirva para controlar la probabilidad de introducir peligros físicos, químicos o biológicos para la inocuidad de los alimentos, sean a través del comportamiento del personal, por deficiencias en la construcción y distribución de los edificios, los suministros de aire, agua, energía y otros servicios, o por mal manejo de residuos.

La organización elabora un único producto para el cual está definido el diagrama de flujo y las etapas de producción; sin embargo, no se evidencian las medidas de control necesarias en estos diagramas ni tampoco la interacción de todas las etapas del proceso, como por ejemplo los procesos contratados externamente, las etapas donde se reprocesan o se reciclan insumos o materias primas. Tampoco se demuestra a donde salen o eliminan los productos finales o intermedios como el agua del lavado.

No se ha establecido un plan HACCP, no hay documentación que lo compruebe. Por lo tanto no se ha realizado un análisis de peligros, ni puntos críticos de control ni acciones correctivas a tomar en caso de que se incumplan los límites y medidas de control.

El sistema de trazabilidad hacia delante y hacia atrás; las operaciones de identificación y seguimiento de materias primas y producto final son sencillas puesto que la organización produce únicamente bajo pedido y envía 
la totalidad de producción al lugar de destino de acuerdo a la orden de pedido que entrega el jefe de ventas.

Al no tener un SGIA implantado la validación, verificación y mejora del sistema resultan insostenibles.

Plan de acción

Ante estos resultados se propone el siguiente plan de acción para mejorar la situación actual de COPROBICH.

Tabla 2. Plan de acción. (Andrade et al., 2017)

\begin{tabular}{|c|c|c|c|c|}
\hline Actividad & Tiempo & Objetivo & Recursos & Responsable \\
\hline $\begin{array}{l}\text { Identificar } \\
\text { el proceso o } \\
\text { problema a } \\
\text { mejorar }\end{array}$ & 2 días & $\begin{array}{l}\text { Incrementar fortalezas, enmendar } \\
\text { debilidades, neutralizar amenazas y } \\
\text { aprovechar oportunidades. }\end{array}$ & Sala de reuniones. & $\begin{array}{l}\text { Líder de inocuidad } \\
\text { Presidente de la } \\
\text { organización } \\
\text { Investigador }\end{array}$ \\
\hline $\begin{array}{l}\text { Identificar las } \\
\text { causas que } \\
\text { originan el } \\
\text { problema }\end{array}$ & 5 días & $\begin{array}{l}\text { Definir los parámetros en los que se } \\
\text { debe trabajar para corregir los } \\
\text { problemas identificados. }\end{array}$ & Test de diagnóstico & $\begin{array}{l}\text { Líder de Inocuidad } \\
\text { Investigador } \\
\text { Auditor interno }\end{array}$ \\
\hline $\begin{array}{l}\text { Definir los } \\
\text { objetivos generales } \\
\text { de la empresa }\end{array}$ & 1 mes & $\begin{array}{l}\text { Definir la condición en que se } \\
\text { solucionarán los problemas, como se } \\
\text { hará, en cuanto tiempo yquien será el } \\
\text { responsable. }\end{array}$ & Sala de reuniones. & $\begin{array}{l}\text { Representante de } \\
\text { cada área de la } \\
\text { organización } \\
\text { Líder de inocuidad } \\
\text { Investigador }\end{array}$ \\
\hline $\begin{array}{l}\text { Definir los } \\
\text { Proyectos y } \\
\text { acciones de mejora }\end{array}$ & 2 meses & $\begin{array}{l}\text { Calendarizar las actividades y las } \\
\text { personas que actuaran en los } \\
\text { proyectos y acciones de mejora. } \\
\text { Definir los recursos necesarios } \\
\text { Establecer posibles obstáculos } \\
\text { Definir indicadores de desempeño e } \\
\text { impacto } \\
\text { Diseñar manuales y levantar registros, } \\
\text { procedimientos e instructivos }\end{array}$ & \begin{tabular}{|l|} 
Sala de \\
reuniones. \\
Rubros necesarios \\
para modificar \\
infraestructura u otros \\
factores que influyen \\
en las no \\
conformidades
\end{tabular} & $\begin{array}{l}\text { Presidente de la } \\
\text { organización } \\
\text { Líder de inocuidad } \\
\end{array}$ \\
\hline $\begin{array}{l}\text { Planear y dar } \\
\text { seguimiento a las } \\
\text { acciones de mejora }\end{array}$ & 3 meses & $\begin{array}{l}\text { Clasificar las acciones de acuerdo a } \\
\text { su prioridad } \\
\text { Validar los manuales, registros, } \\
\text { procedimientos e instructivos } \\
\text { redactados } \\
\text { Capacitación y puesta en marcha de } \\
\text { aquellas acciones prioritarias } \\
\text { Establecer planes de mejora }\end{array}$ & $\begin{array}{l}\text { Sala de reuniones } \\
\text { Representantes de la } \\
\text { empresa } \\
\text { Investigador }\end{array}$ & Líder de inocuidad \\
\hline
\end{tabular}

\section{Estrategias aplicadas en COPROBICH}

\section{Requisitos Generales}

Estrategia: A través del desarrollo de este trabajo de investigación se definió el objeto y alcance del SGIA diseñado para COPROBICH, el mismo que contempla la elaboración, documentación, aprobación y socialización de los procedimientos y registros necesarios para alcanzar una mejora continua en los principales procesos que están inmersos en la calidad final del producto: 
solicitudes de venta, proveedores calificados, recepción de materia prima e insumos, producción y transporte de la quinua procesada.

Se identificaron y evaluaron los peligros relacionados con la inocuidad de los alimentos para asegurarse de que no dañen al consumidor. Para esto se comunicó a todos los miembros de la cadena alimentaria la información concerniente al desarrollo, la futura implementación y la actualización del SGIA.

\section{Requisitos de la documentación}

Estrategia: En el momento de la intervención la organización no contaba con ningún tipo de documento, para lo cual se desarrolló toda la documentación necesaria (Procedimientos, manuales, registros, POE y POES) que soporte el modelo de SGIA diseñado para COPROBICH. Este listado de documentos y sus respectivos códigos se puede evidenciar en el Documento Matriz General de Documentación.

Se incluye también la forma en que se aprobarán los documentos antes de su emisión, y quién tiene la autoridad de aprobar, revisar y actualizar los mismos.

\section{Compromiso de la dirección}

Estrategia: La organización, al aceptar el Diseño de un SGIA para la planta de procesamiento de quinua, pone en evidencia el compromiso de la dirección en el desarrollo e implementación del SGIA. De igual forma, la alta dirección afianza su comunicación interna para hacer saber a todos sus colaboradores la importancia de cumplir con el Sistema de inocuidad.

\section{Política de la inocuidad de los alimentos}

Estrategia: Se apoyó la declaración documentada de una política de inocuidad de alimentos y objetivos relacionados a la misma. COPROBICH posee su política de calidad impulsada por la administración e impartida a todos quienes conforman la empresa, la cual establece básicamente la responsabilidad de entregar al consumidor alimentos y servicios de excelente calidad y seguridad alimentaria apoyada en los principios de higiene de los alimentos; esta comprende:

v. Otorgar la máxima garantía de inocuidad de los alimentos que elaboramos, protegiendo la salud de los consumidores que día a día depositan su confianza en los productos.

vi. Orientación y satisfacción de las necesidades y expectativas identificadas en los clientes por medio de un servicio de calidad eficaz que incluya: alto nivel nutricional y producción orgánica e industrialización segura. 
vii. El mejoramiento de la infraestructura que permita el cumplimiento de requisitos, una mejora continua de los procesos productivos, incremente la rentabilidad y extienda el prestigio de la empresa en territorio extranjero.

viii. Formar, motivar y responsabilizar al personal en su puesto de trabajo ya que estamos conscientes que se debe invertir en el talento humano para la participación activa en la mejora continua, de forma que se aplique un autocontrol en la calidad del trabajo que realizan.

ix. Compromiso de protección y preservación del medio ambiente.

\section{Planificación del sistema de gestión de la inocuidad de alimentos}

Estrategia: Se llevó a cabo la planificación del SGIA para cumplir con los requisitos de la norma y los objetivos de inocuidad.

\section{Responsabilidad y autoridad}

Estrategia: Se definió la responsabilidad y autoridad de cada uno de los miembros de la cadena alimentaria y fueron socializadas dentro de la organización para que todos identifiquen al personal para iniciar y registrar acciones del SGIA.

\section{Líder del equipo de la inocuidad de los alimentos}

Estrategia: Se designó como líder de inocuidad a la Ing. Ximena Chávez quien inicia sus funciones como Jefe de Producción en la organización. Además, se definió un equipo de inocuidad con la responsabilidad y autoridad para dirigir al equipo de inocuidad y organizar su trabajo. Se dieron programas de capacitación para asegurar la formación y educación pertinente de los miembros del equipo de inocuidad.

\section{Comunicación}

Estrategia: La comunicación externa es eficaz; sin embargo, se diseñaron registros y procedimientos para evidenciar la eficacia del sistema de comunicación. Se cuenta con disposiciones eficaces para comunicarse con los proveedores y contratistas, clientes y consumidores, auditorías legales y reglamentarias y organizaciones que afectan o serán afectadas por la eficacia o actualización del SGIA y para usarlas posteriormente como información de entrada para posteriores revisiones y verificaciones del sistema. La comunicación interna tiene ahora un protocolo a seguir para manejar la información dentro de la organización.

\section{Preparación y respuesta a emergencias}

Estrategia: Se diseñó un procedimiento donde se indica cómo se deben gestionar las potenciales situaciones de emergencia y accidentes que pueden 
afectar la inocuidad de los alimentos, cuáles son estas situaciones y se incluye un simulacro.

\section{Revisión por la dirección}

Estrategia: Se estableció un intervalo de tiempo para la revisión del SGIA por parte de la alta dirección.

\section{Provisión de los recursos}

Estrategia: La organización se comprometió a proveer los recursos necesarios para establecer, implementar, mantener y actualizar el SGIA.

\section{Recursos humanos}

Estrategia: Se evaluaron las competencias del equipo de inocuidad de alimentos y demás personal relacionado con la inocuidad de alimentos, para poder definir un equipo de inocuidad de alimentos. Se desarrollaron procedimientos y registros para la competencia, toma de conciencia y formación del personal. Se realizó también un programa de formación del personal

\section{Infraestructura}

Estrategia: La organización, a partir de la información entregada después de la auditoría de diagnóstico, se comprometió a proporcionar los recursos adecuados para así entregar los insumos necesarios para establecer y mantener la infraestructura necesaria para asegurar la inocuidad de los alimentos. Al ser una organización sin fines de lucro, su presupuesto es reducido, por lo que se comprometió también a gestionar la obtención de recursos económicos que permitan modificar la infraestructura de edificios e instalaciones y adquirir equipospara el control de los puntos críticos de control identificados. Después de algunos meses se obtuvo el financiamiento para las modificaciones respectivas por parte del Instituto Nacional de Economía Popular y Solidaria.

\section{Ambiente de trabajo}

Estrategia: La organización está dispuesta a proporcionar los recursos adecuados para establecer y mantener el ambiente de trabajo necesario para asegurar la inocuidad de los alimentos.

\section{Planificación y realización de productos inocuos}

Estrategia: La organización tomará en cuenta los parámetros sugeridos de modo que se garanticen alimentos inocuos durante la planificación de la producción que se elabore cuando llegue el pedido al área de ventas, considerando que la planta sólo produce bajo pedido. 


\section{Programas Prerrequisito (PPR)}

Estrategia: Se estandarizaron los procesos operativos y los procesos operativos de sanitización y se diseñaron registros que permitan ayudar a controlar los niveles de peligro (químico, físico o biológico) relacionado con la inocuidad de los alimentos en el producto y en el ambiente donde se elabora. Con el apoyo del equipo de inocuidad se aprobaron los PPR validando que estos estén acorde al tamaño y al tipo de operación y a la naturaleza de los productos que se elaboran, así como el cumplimiento de las características de construcción y distribución de los edificios y de las instalaciones de aire, agua, energía y otros servicios de apoyo incluyendo la eliminación de desechos.

\section{Pasos Preliminares para permitir el análisis de peligros}

Estrategia: Se validó el diagrama de flujo existente y adicionalmente se incorporaron en el mismo las medidas de control, la secuencia e interacción de todas las etapas del proceso, los procesos contratados, las etapas donde se incorpora al flujo materias primas, ingredientes y productos intermedios, y las etapas donde se reprocesa.

\section{Análisis de riesgos}

Estrategia: Se desarrolló un análisis de peligros, para identificar los peligros físicos, químicos y biológicos de cada una de las etapas y establecer los niveles aceptables de peligro en el producto final, tomando en cuenta la legislación, los requisitos del cliente y el uso previsto.

\section{Pasos preliminares para permitir el análisis de peligros}

Estrategia: Evaluamos los peligros de acuerdo a su posible severidad y probabilidad para determinar si su eliminación o reducción a niveles aceptables es esencial para la producción de un alimento inocuo. Se seleccionaron y evaluaron las medidas de control para los peligros identificados permitiéndose identificar los PPR operativos y documentarlos.

\section{Establecimiento de los programas prerrequisito operativos (PPR operativos)}

Estrategia: Se documentaron los PPR operativos, los cuales incluyen procedimientos de seguimiento que demuestren que los PPR operativos están implementados, correcciones y acciones correctivas en caso de que existan desviaciones, responsabilidades y autoridades.

La vigilancia de los peligros asociados a la inocuidad dentro del proceso operativo inicia con la evaluación y control en el momento de la recepción de la materia prima (quinua) en la planta de producción, el cual partirá de la valoración de la humedad y la presencia de sustancias extrañas a la naturaleza del alimento, Dicho control es registrado en el documento 
RPCCOPROBICH0701-01: Registro de Proveedores de Materia Prima y es controlado y verificado por la Jefe de Sistema de Gestión de calidad.

$$
\text { Procedimiento de desaponificación de quinua }
$$

(PCOCOPROBICHQUIN). Dentro de estos se contemplan los Instructivos de Desaponificación de Quinua y el Instructivo de Empacado de quinua, los cuales describen los procedimientos para llevar a cabo esta operación y las acciones correctivas en caso de no cumplir con las especificaciones establecidas para el producto final, en cuyo caso no podrá comercializarse si presenta algún defecto de proceso. Luego de que la quinua haya sido desaponificada y secada se aplica el procedimiento PCCOPROBICH0706: Gestión de Despacho que tiene por objetivo indicar los diferentes pasos para el despacho de producto terminado que elabora COPROBICH.

\section{Establecimiento del plan HACCP}

Estrategia: Se estableció y documentó el Plan HACCP; en este se contemplaron los siguientes aspectos:

Peligros relacionados con la inocuidad de los alimentos a controlar en los PCC, Medidas de control, límites críticos, Procedimientos de seguimiento, correcciones y acciones correctivas a tomar si se superan los límites críticos, responsabilidades y autoridades, identificación de los puntos críticos de control (PCC), si se tienen identificados los puntos críticos de control para las medidas de control identificadas, si se especifican las correcciones planificadas y las acciones correctivas a tomar cuando se superan los límites críticos, y finalmente si se cuenta con un procedimiento para la correcta manipulación de los productos potencialmente no inocuos.

22. Actualización de la información preliminar y de los documentos que especifican los PPR y el plan HACCP

Estrategia: Una vez determinados los programas de prerrequisitos operacionales y el plan HACCP, la organización se comprometerá a mantener actualizada la información referente a:

- Características del producto.

- Uso previsto.

- Los diagramas de flujo

- Las etapas del proceso.

- Las medidas de control.

\section{Planificación de la verificación}

Estrategia: Junto con el líder de inocuidad se planificó la verificación del sistema una vez que este haya sido implementado, de manera que se revisará anualmente el propósito, método, frecuencia y responsabilidad para las actividades de verificación. 


\section{Sistema de trazabilidad}

Estrategia: Se cuenta con un sistema de trazabilidad que identifica los lotes de materia prima, registros de procesamiento y entrega. La organización maneja y registra su sistema de trazabilidad eficientemente puesto que al trabajar bajo pedido la planta puede fácilmente identificar a los proveedores que entregaron la producción para el pedido a despachar y a su vez puede fácilmente dar seguimiento al producto terminado puesto que se envía en un solo embargue el total de producción de acuerdo al país de destino del pedido.

\section{Control de no conformidades}

Estrategia: Las acciones correctivas relacionadas a los procesos de la empresa son identificadas con el código PCCOPROBICH 0803 (Acciones preventivas, correctivas y de mejora); estas al ser detectadas deben ser registradas en el documento RPCCOPROBICH0708-01. La finalidad del procedimiento es asegurar la calidad e inocuidad de los productos mediante la inspección y revisión de los registros de calidad del producto, para que en caso de incumplimiento de requisitos se proceda a su reprocesamiento o eliminación según el tipo de no conformidad del producto.

En el caso particular de la quinua, el reproceso de productos es únicamente válido cuando el producto se encuentra mal etiquetado, procediéndose a devolver el producto a producción para que se etiquete correctamente y luego reingrese a la bodega de PT para su comercialización. En el caso de que los residuales de saponinas sean demasiado altos se procede a preparar una parada de quinua para ser nuevamente lavada; y en el caso de que la humedad de la quinua sea mayor al $10 \%$ se procederá a colocar nuevamente en las camas de secado.

Esto se detalla en los instructivos: IPCCOPROBICH0708-01 (Acciones correctivas en el secado y desaponificación de quinua)y IPCCOPROBICH0708-02 (Acciones Correctivas en Despachos). La alta dirección debe garantizar que el retiro de un lote de producto identificado como no inocuo sea rápido y fácil, para lo cual designará a los responsables que tendrán la autoridad para iniciar una retirada del producto; estos deberán actuar guiados por el procedimiento descrito en el documento PCCOPROBICH 0709. (Retiro de Producto), actividad que se facilitará puesto que todos los productos están debidamente identificados de tal manera que haga más fácil su rastreabilidad (PCCOPROBICH 0707).

El Director Administrativo Financiero dispone de una base de datos con los nombres de los contactos, dirección y teléfonos que pueden hacer posible la comunicación inmediata. El JLV mantiene los registros del producto despachado y su lugar de destino para la rápida localización de los lotes distribuidos. 
26. Validación, verificación y mejora del sistema de gestión de inocuidad de alimentos, generalidades

Estrategia: Se estableció que la validación de las medidas de control y/o las combinaciones de medidas de control deben ser planificadas e implementadas por el equipo de la inocuidad de los alimentos, de manera que estas acciones permitan verificar y mejorar el sistema de gestión de la inocuidad de los alimentos.

\section{Validación de las combinaciones de medidas de control}

Estrategia: Luego de definidas o modificadas las medidas de control y previo a su inclusión en los PPR operativos y a su implementación en el proceso, la organización deberá validar la capacidad de estas medidas de vigilancia sobre el control de los peligros relacionados con la inocuidad y que su eficacia al combinarse garantice el control de los peligros para obtener productos finales que cumplan con los niveles de aceptación definidos.

\section{Control de seguimiento y medición}

Estrategia: La organización deberá tener formas de demostrar que los métodos y equipos de seguimiento y medición especificados facilitan el desarrollo de los procedimientos de seguimiento y medición, así como también que cuando resulte necesario los equipos sean calibrados o verificados en intervalos específicos con patrones comparativos previo a su utilización. COPROBICH cuenta con un Plan Anual de Mantenimiento, así como con instructivos y registros de las actividades de control y medición que se llevan a cabo en la organización. PCCOPROBICH 0703.(OPERACION Y MANTENIMIENTO PREVENTIVO DE EQUIPOS E INSTALACIONES).

\section{Verificación del sistema de gestión de la inocuidad de los alimentos}

Estrategia: Los mecanismos de supervisión del sistema son obligatorios y deben ser revisados y actualizados según el "Procedimiento de Verificación y Validación del Sistema HACCP". La Presidencia de COPROBICH se compromete a mejorar continuamente el sistema de gestión de la empresa, a través de la comunicación de los objetivos, la evaluación de los recursos y la revisión periódica de los procesos.

La organización cuenta con un procedimiento PCCOPROBICH 0802 (AUDITORIAS INTERNAS) para describir la manera de planificar y ejecutar las auditorías internas al Sistema de Calidad vigente con base al Manual, los Procedimientos e Instructivos de COPROBICH.

El líder de inocuidad elabora el programa anual de auditorías para que sea aprobado por el director administrativo financiero de la corporación. Estas deberán realizarse mensualmente y si por motivos extraordinarios no se 
cumple el programa anual de auditorías internas, éste podrá ser modificado por autorización del responsable de aprobar este tipo de documentación. Todos estos procesos serán documentados en el RPCCOPROBICH 0802- 01.

Se planificarán también auditorías externas que permitirán que la empresa y el personal reciban un entrenamiento para la acreditación y certificación global del sistema de gestión de inocuidad de los alimentos.

Las observaciones y no conformidades detectadas durante las auditorías se registrarán en el documento RPCCOPROBICH0802-06: (Informe de no conformidades e implantación de acciones correctivas) y se entregarán en un informe final a la Dirección para su inmediata revisión. Cabe resaltar que las auditorías además de controlar los procedimientos de la empresa también sirven como instrumentos de mejora continua para el sistema de gestión.

El equipo de la inocuidad de los alimentos debe evaluar los resultados individuales de la verificación que cada responsable presente de acuerdo a lo planificado e informar a la dirección si existe conformidad con lo planificado en el sistema de gestión; caso contrario debe tomar las medidas necesarias para cumplir con los objetivos proyectados por la empresa. Se deberá realizar la revisión de los procedimientos existentes, las rutas de comunicación, los PPR operativos establecidos y el plan HACCP.

El auditor líder prepara el informe resumido de la auditoría según el RPCCOPROBICH0802- 07 y lo expone en la reunión final que está integrada por los mismos miembros que asistieron a la reunión inicial. El informe final definitivo de la auditoría lo entrega el auditor líder al presidente, en un plazo máximo de 2 días después de realizada la auditoría. Este informe debe contener los puntos indicados en el RPCCOPROBICH 0802-07.

En base al informe, el presidente de la corporación puede recomendar modificación(es), solución(es) de las no conformidades. El líder lleva un control de la eliminación de las no conformidades en el RPCCOPROBICH 0802-08 y mantiene un archivo de los informes finales de auditoríaa y sus documentos de soporte.

La alta dirección está consciente de que debe mejorar continuamente la eficacia de su sistema de inocuidad de los alimentos, para lo cual utiliza su sistema de comunicación interno y externos, revisiones anuales de procedimientos, registros y manuales con la alta dirección y mensuales con los responsables de cada proceso, auditorías internas, revisiones de los resultados de verificaciones, validaciones de las medidas de control, acciones correctivas y reclamos y la actualización continua del sistema de gestión; todo esto con la finalidad de introducir mejoras que permitan elevar el nivel de calidad de la organización. 


\section{Resultados de la Auditoría después del diseño SGIA}

口Puntajes máximos a obtener [Puntaje Obtenido

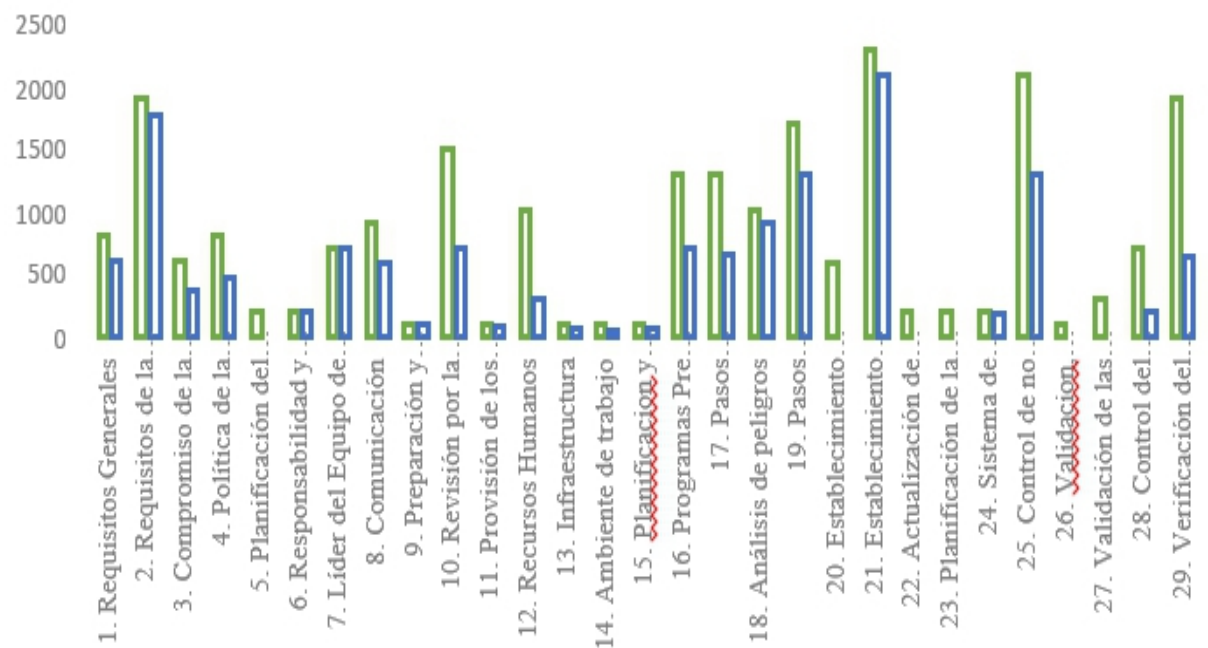

Figura 2. Esquema comparativo de los resultados de auditoría posterior al diseño del SGIA

En la figura 2 se muestra un esquema comparativo de los resultados de la auditoría aplicada después del Diseño del Modelo de Gestión de la Inocuidad de los Alimentos. Se puede observar cómo la organización muestra una mejora significativa en cada uno de los ítems considerados dentro de la Norma ISO 22000:2005.

\section{Conclusiones}

Se diseñó un Sistema de Gestión de Inocuidad Alimentaria bajo la Norma ISO 22000:2005 para la planta procesadora de quinua de la Corporación de Productores y Comercializadores Orgánicos Bio Taita Chimborazo, COPROBICH a través de la identificación de todos los peligros que se asocian con la inocuidad de un alimento y que pueden aparecer en cualquier etapa del procesamiento de la quinua. Se trabajó para ayudar a la corporación a comprometer a todos los miembros de la cadena alimentaria en el cumplimiento de las políticas y de los objetivos de calidad e inocuidad que se plantearon mediante la aplicación de procedimientos, registros y manuales que le permitan a la organización sostener de forma documentada la calidad que oferta su producto y facilitar la comercialización de este cultivo andino en nuevos mercados a nivel internacional.

El diseño del Sistema de Gestión de Inocuidad partió de la realización de un diagnóstico de los procesos productivos, desde la recepción de la materia 
prima hasta la entrega del producto terminado a través de la aplicación de un formato para la evaluación del estado sanitario del establecimiento según la Norma Internacional ISO 22000:2005. El resultado obtenido refleja un $88 \%$ de no conformidades, por lo que se concluye que la organización requiere el desarrollo de un sistema de Gestión de Inocuidad de los alimentos de forma inmediata. Para esto se diseñó y documentó la información necesaria para sustentar un modelo de Sistema de Gestión de la Inocuidad Alimentaria aplicable a la realidad COPROBICH, modelo que demostró el impacto positivo que tuvo a través de la aplicación posterior del mismo cuestionario de diagnóstico en el que se redujo el porcentaje de no conformidades a un $48 \%$.

En resumen, se planteó un modelo de sistema de gestión de calidad que al ser validado e implementado garantice la inocuidad de los productos elaborados en la planta de quinua de la Corporación de Productores y Comercializadores Orgánicos Bio Taita Chimborazo (COPROBICH).

\section{References:}

1. Aldana Martínez, A. M. (Julio de 2010). Implementación Del Programa De Buenas Prácticas De Manufactura, En El Departamento De Producción De Café Dresden. Guatemala: Universidad de San Carlos de Guatemala.

2. Álvarez, R. F. (2012). Importancia de la gestión de la inocuidad alimentaria e instrumentos para su implementación en la empresa. Éxito Empresarial, 1-5.

3. Arcsa, M.D. (2015). Normativa Técnica Sanitaria Sobre Prácticas Correctas De Higiene Para Establecimientos Procesadores De Alimentos Categorizados Como Artesanales Y Organizaciones Del Sistema De Economía Popular Y Solidaria. Resolución Arcsa-De-0572015-GGG. Ecuador.

4. Briones Vinueza, K. (2009). Preparación Documenetal de los Requerimientos de la Norma ISO 22000:2005 a ser aplicados en una Empacadora de Camarón. Guayaquil, Guayas, Ecuador.

5. Carranza Monroy, A. L. \& Carranza Monrroy, F. (Noviembre de 2012). Sistema de Gestión de la Calidad ISO-22000 Para la Sociedad Cooperativa Yuntathui de R.L. El Salvador, El Salvador.

6. Chimborazo, C. d. (s.f.). Obtenido de http://es.coprobich.com/quienessomos/ Comisión del Codex Alimentarius, P. C. (1999). Codex Alimentarius - Higiene de los Alimentos. En C. Alimentarius, Requisitos Generales (Higiene de los Alimentos).

7. COPROBICH (2015). Corporación de Productores y Comercializadores Orgánicos Bio Taita Chimborazo. Obtenido de http://es.coprobich.com/quienes-somos/

8. Cossio, J. (Octubre de 2005). Fasiculo 1- Preparación de suelo. 
In:PROINPA Y FAUTAPO. Serie de Módulos Publicados en Sistemas de producción Sostenible en el Cultivo de la Quinua: Módulo 2. Manejo agronómico de la Quinua Orgánica.

9. Fundación PROINPA, Fundación AUTAPO. La Paz, Bolivia.

10. Cuenca Chacon, M.L. \& Montenegro Aguirre, A.D. (2004). Plan de Negocios para la Creación de una Empresa dedicada a la Elaboración de Malteadas a base de Quinua en la Ciudad de Bogotá D.C. Bogotá, Colombia.

11. Decreto Ejecutivo 756, R. O.-m.-2. (17 de Mayo de 2011). REGLAMENTO GENERAL A LA LEY DEL SISTEMA ECUATORIANO DE LA CALIDAD. Quito, Ecuador.

12. FAO, O.A. (Julio de 2011). La Quinua: Cultivo milenario para contribuir a la seguridad alimentaria mundial.

13. Fuentes, F.C. (Octubre de 2005). Implementación de un Plan de Buenas Prácticas de Manufactura en el Proceso de Empaque del azúcar. Guatemala: Universidad de San Carlos de Guatemala.

14. García Campana, M. F. (2007). Propuesta de Diseño del sistema de Gestión de la Calidad en Eléctricos Nacionales (ELENTRAC), según la Norma ISO 9001:2000. Quito, Pichincha, Ecuador.

15. Guerrero Vargas, M. (Julio de 2005). La Seguridad Alimentaria, un Derecho Fundamental del Ser Humano. Quito, Ecuador.

16. Instituto de Formación Integral. Antonio Limón. (2006). Guía para la Aplicación de la Norma UNE -EN-ISO 22000. Sistemas de Gestión de la Inocuidad de los Alimentos. España.

17. International Organization for Standardization (24 de Noviembre de 2015). International Organization for Standardization. Obtenido de http://www.iso.org/iso/home/standards/managementstandards/iso22000.htm

18. Iñaki (2007). La integración de sistemas de gestión basados en estándares internacionales: resultados de un estudio empírico realizado en la Capv1, s/n. Revista de Dirección de Administración de Empresas, 157.

19. ISO 22000 (2005). Sistemas de gestión de la inocuidad de los alimentos - Requisitos para cualquier organización en la cadena alimentaria.

20. Maukonen, T. (2009). Producción Alimentaria. Programa de las Naciones Unidas para el Medio Ambiente.

21. Mauricio, A. M. (Julio de 2010). Implementación Del Programa De Buenas Prácticas De Manufactura, En El Departamento De Producción De Café Dresden. Guatemala: Universidad de San Carlos de Guatemala.

22. Meyhuay, M. \&Agroindustral, I.D. (Junio de 1997). Quinua: 
Operaciones de Poscosecha.

23. Mina Chalá, D.F. (2014). Evaluación Agronómica de Líneas F5 de Quinua (Chenopodiumquinoa). Quito, Ecuador.

24. Ogalla, S. F. (2005). Sistema de Gestion: Una guía practica. España: Díaz de Santos.

25. Organización Internacional de Normalización (2008). Norma 9000. O.I.Normalización.

26. Palma Terán, D. A. (Abril de 2011). Estudio Para La Implementación De Buenas Prácticas De Manufactura En Los Servicios De Alimentación Del Hotel Río Amazonas. Quito, Ecuador: Escuela Politécnica Nacional.

27. Pilatti, H. (2007). Higiene e Inocuidad de los Alimentos: Procedimientos Operativos Estandarizados de Saneamiento (POES). El Boletín del Inspector Bromatológico $N^{\circ}$ 9, INSTITUTO NACIONAL DE ALIMENTOS.

28. Ramírez Melo, C. \& Sánchez Herrero, M. C. (2006). Implementación del Sistema de Gestión de Calidad, en base a la norma ISO 9001:2000. Hidalgo, México.

29. RiofrioAlbuja, M. F. \& Moran, A.W. (2010). Diseño del Sistema de Gestión de Inocuidad Alimentaria ISO 22000-2005 en una Empresa Elaboradora de Premezclas de Panificación. Guayaquil, Ecuador.

30. RiofríoAlbuja, M. F. \& Moran Arcentales, W. R. (2009). Diseño del Sistema de Gestión de Inocuidad Alimentaria ISO 22000:2005 en una Empresa Elaboradora de Premezclas de Panificación. Guayaquil, Ecuador.

31. Salud, O. P. (s.f.). Organización Panamerica de la Salud. Obtenido de http://www.paho.org/hq/?lang=es

32. Salud, O. P. (s.f.). Organización Panamericana de la Salud PAHO/WHO. Obtenido de Principios generales de Higiene de los Alimentos del Codex: http://www.paho.org/hq/index.php?option=com_content\&view=articl e\&id=10562\% 3A2015-principios-generales-de-higiene-de-losalimentos-del- codex\&catid=7676\%3Acodexalimentarius \&Itemid=41271 \&lang=es

33. Standardization, I.O. (24 de Noviembre de 2015). International Organization for Standardization. Obtenido de http://www.iso.org/iso/home/standards/managementstandards/iso22000.htm

34. Valhondo, J. (2013). Dirección de Operaciones. Introducción. Cataluña, España.

35. Van, C. (2005). Seminario Taller 'Los Sistemas de Gestión en la Industria Ecuatoriana“. 
36. DECAB, Bioprocesos.

37. Wong López, N. E. (Julio de 2014). Diseño de un Sistema de Gestión de la Calidad y su Implementación, Primera Etapa, en un Laboratorio de la Dirección Nacional de Investigación y Aplicaciones Núcleares DNIAN. Quito, Ecuador. 